\title{
Ejection Fraction, Qualitative
}

National Cancer Institute

\section{Source}

National Cancer Institute. Ejection Fraction, Qualitative. NCI Thesaurus. Code C80418.

A descriptive adjective assigned to the computed amount of blood ejected during a ventricular contraction of the heart. 